the direction of the magnetic field of the photon, so that $\overrightarrow{\mathrm{k}}_{E}$ is a vector and $\overrightarrow{\mathrm{k}}_{M}$ a pseudovector.

${ }^{8}$ The only $\pi^{+} \pi^{-} \gamma$ matrix element that approximates the $\pi^{+} \pi^{-}$spectrum is $J^{P}=2^{-}$for $C=-1$; however, the angular distribution predicted by this matrix element does not agree with the data.

${ }^{9}$ For dipole transitions to $\rho \gamma$, only $J=0,1$, or 2 is possible.

${ }^{10}$ Reference 3 gives a more detailed argument in regards to determining the $G$-parity of the meson.

${ }^{11}$ In the current experiment we have also taken about 80000 pictures of $2.63-\mathrm{BeV} / c K^{-}$in deuterium. The (expected) absence of an effect in this sample will be significant enough to confirm the isotopic-spin assignment.

${ }^{12}$ The $231 \gamma$ and two $2 \gamma$ events contain 17 conversion pairs, eight Compton electrons, and two Dalitz pairs.

${ }^{13} \mathrm{An}$ approximate detection efficiency was determined from a sample of $199 \Lambda \omega$ events, which yielded 13 "visible" gammas, as well as from the two $\pi^{+} \pi^{-} \gamma_{V}$ in the $42 \pi^{+} \pi^{-} \gamma$ events. We have ignored differences in the gamma energy spectra of the various samples in this zero-order correction.

${ }^{14}$ The decay rate into $\pi^{+} \pi^{-} \gamma$ is estimated at $\sim 5 \%$ by L. M. Brown and H. Faier, Phys. Rev. Letters 13 , 73 (1964), which is lower than the $\sim 20 \%$ rate observed. They assume that the $T J P G=00^{++}$, two-pion enhancement (" $\sigma$ ") plays an important part in the $\eta 2 \pi$-decay mode. Without this enhancement, the $\pi^{+} \pi-\gamma$ decay would represent a larger fraction of the total decay rate. The $\pi^{+} \pi^{-}$spectrum associated with the $\eta 2 \pi-$ decay mode does not lend support to the " $\sigma$ " hypothesis, unless the mass of the " $\sigma$ " is lowered from the usual $\sim 400-\mathrm{MeV}$ value down to $\sim 350 \mathrm{MeV}$.

${ }^{15}$ With the improved resolution of the $\Lambda \pi^{+} \pi^{-} \eta$ fits and the larger sample, our best value of the mass is $M=958 \pm 1 \mathrm{MeV}$ and of the width is $\Gamma \leqslant 7 \mathrm{MeV}$.

${ }^{16} \mathrm{M}$. Gell-Mann, California Institute of Technology Synchroton Laboratory Internal Report No. CTSL-20, 1961 (unpublished); M. Gell-Mann, Phys. Rev. 125, 1067 (1962); J. Schwinger, Phys. Rev. Letters $\underline{12}$, 237 (1964); A. Pais, Phys. Rev. Letters 12, 634 (1964); C. Becchi and G. Morpurgo, Phys. Rev. Letters $\underline{13}, 110$ (1964).

\title{
SEARCH FOR FRACTIONALLY CHARGED PARTICLES PRODUCED BY $27.5-\mathrm{GeV} / c$ PROTONS*
}

\author{
W. Blum, ${ }^{\dagger}$ S. Brandt, V. T. Cocconi, O. Czyzewski, ${ }^{\ddagger}$ J. Danysz, ${ }^{\ddagger}$ M. Jobes, ${ }^{\S}$ \\ G. Kellner, "D. Miller,** D. R. O. Morrison, W. Neale, ${ }^{* *}$ and J. G. Rushbrooke ${ }^{\dagger \dagger}$ \\ CERN, Geneva, Switzerland
}

(Received 4 August 1964)

A bubble chamber experiment has been performed to search for fractionally charged particles, following the suggestions by Gell-Mann ${ }^{1}$ and Zweig" that long-lived particles, "quarks" or "aces," might exist with electric charge $\pm \frac{1}{3}$ and $\pm \frac{2}{3}$ of that of the electron, and with masses small enough to be produced by present-day accelerators. The result of the experiment may be summarized with the statement that longlived quarks of charge between 0.2 and $0.7 e$ are not produced in nucleon-nucleon interactions with a cross section greater than about $10^{-35}$ $\mathrm{cm}^{2}$, if their mass is $\leqslant 2.5 \mathrm{GeV}$, and about $10^{-32}$ $\mathrm{cm}^{2}$, if their mass is $\leqslant 4.0 \mathrm{GeV}$.

The 27.5-GeV/c circulating beam of the CERN proton synchrotron was allowed to strike an internal copper target. A secondary beam of negative particles with momentum $p_{\text {app }}=20 \mathrm{GeV} / c$ emitted at $76 \pm 6 \mathrm{mrad}$ was selected and passed through the Saclay $81-\mathrm{cm}$ hydrogen bubble chamber located $140 \mathrm{~m}$ from the target in a magnetic field of $20.6 \mathrm{kG}$. If particles, $q$, with fractional charge, $z$, were produced at the target in reactions, the simplest of which is

$$
p+N \rightarrow N+N+q+\bar{q},
$$

their momentum would be $p_{\mathrm{app}} / z$, i.e., $6.7 \mathrm{GeV} /$ $c$ for $z=\frac{1}{3} e$ and $13.4 \mathrm{GeV} / c$ for $z=\frac{2}{3} e$. The upper limit of their mass, if produced on free nucleons at rest, would be $2.54 \mathrm{GeV}$ and $2.48 \mathrm{GeV}$, respectively.

The chamber was operated in conditions such that pions produced tracks with 20 bubbles $/ \mathrm{cm}$, on the average. As the bubble density is proportional to $z^{2}$, a quark of $z=\frac{1}{3} e$ would produce a track with 2-3 bubbles $/ \mathrm{cm}$ and a quark of $z=\frac{2}{3} e$ about 10 bubbles $/ \mathrm{cm}$.

A search for fractionally charged particles using a hydrogen bubble chamber, as described, meets with two systematic difficulties: (a) If no tracks of subnormal bubble density are observed, one has no information on the scanning efficiency; and (b) the observation of a track of subnormal bubble density is not sufficient to prove the existence of a particle with fractional charge. In fact, conditions may occur in which a particle of normal charge leaves in the chamber a track with bubble density lower than normal. Ideally, the beam, of short duration $(0.4$ msec in our case), is made to enter the chamber during the period of the expansion cycle corresponding to the plateau of minimum pres- 
sure and full sensitivity. However, depending on the target operation and on the structure of the beam, it may occasionally happen that a particle hits the target some time before the main beam is deflected onto it. One of its secondaries may then enter the chamber before this has reached full sensitivity, consequently leaving a track with bubble density lower than normal. An "early track" will have bubbles, in general, bigger in diameter than a normal track, as its bubbles have had longer growth time. However, since on the film the bubble images are mainly diffraction spots, early tracks are not easily distinguishable from normal tracks in a hydrogen bubble chamber.

To overcome these two difficulties the following procedure has been used: Simultaneously with each bubble chamber photograph, a picture was taken of three oscilloscope traces displaying the time relationships of (a) the pulse triggering the flash voltage, (b) the chamber pressure curve, and (c) the counter pulse caused by each particle entering the chamber. By correlating the bubble chamber and the oscilloscope photographs, one could thus determine in each case whether or not a track observed to have subnormal bubble density corresponded to an "early" normal particle.

In 14000 pictures with, on the average, 11 tracks/picture, 19 tracks were found with bubble density lower than normal. Mean gap-length measurements made on these tracks as well as on a sample of normal beam tracks indicated that these "quarklike tracks" had bubble densities ranging from $\approx 1 / 50$ to $\frac{1}{2}$ of normal, a range that included the densities expected for quarks.

However, for every one of these tracks, the corresponding oscilloscope record revealed the occurrence of an "early pulse," corresponding to a particle of normal charge that had entered the chamber 1-2 msec before the correct beam time $(t=0)$. Particles entering the chamber more than $2 \mathrm{msec}$ before $t=0$ did not leave observable tracks as the chamber was too insensitive, while particles arriving less than 1.0 msec before $t=0$ generally found the chamber sensitive enough to leave tracks indistinguishable from normal.

It is important to note that not only every track observed with subnormal bubble density corresponded to an early pulse, but that, vice versa, every pulse in the interval $1<t<2$ msec corresponded to a track of subnormal bubble density actually observed in the scan.
One may therefore conclude that: (a) The scanning efficiency for "quarklike" tracks, and hence for possible quarks, can be taken as $100 \%$; and (b) no track was observed that had to be attributed to a particle of subnormal charge.

This sets an upper limit on the production cross section of the fractionally charged particles that could have been observed.

The solid angle $\Delta \Omega$ and the bite of apparent momentum $\Delta p_{\text {app }}$ accepted at the detector were such that $\Delta \Omega \Delta p_{\text {app }}=4 \times 10^{-6} \mathrm{sr} \mathrm{GeV} / c$. The protons hitting the target were $25 \%$ of a circulating beam of $6 \times 10^{11}$ protons/pulse, which interacted in the target with $\approx 50 \%$ efficiency. The differential cross section corresponding to the observation of one fractionally charged particle in $1.4 \times 10^{4}$ pulses is then

$$
\begin{aligned}
\left(\frac{d^{2} \sigma}{d p d \Omega}\right)_{\mathrm{lab}} & =\frac{\sigma_{\text {tot }}}{7.5 \times 10^{10} \times 1.4 \times 10^{4} \times 4 \times 10^{-6}} \\
& =9.5 \times 10^{-36} \frac{\mathrm{cm}^{2}}{\mathrm{sr} \mathrm{GeV} / c}
\end{aligned}
$$

with $\sigma_{\text {tot }}=40 \mathrm{mb}$.

Estimates of the upper limit on the total cross section have been obtained assuming isotropic production of quarks in the c.m. system and momentum distribution according to the phase space for the four-body reaction (1). For quarks of charge $\frac{1}{3} e$ the result is

$$
\begin{aligned}
\sigma_{\text {tot }} & \leqslant 6.5 \times 10^{-35} \mathrm{~cm}^{2} \text { if the quark mass is } 1 \mathrm{GeV} \\
& \leqslant 2.0 \times 10^{-35} \mathrm{~cm}^{2} \text { if the quark mass is } 2 \mathrm{GeV} .
\end{aligned}
$$

Similar values are found for quarks of charge $\frac{2}{3} e$.

Values of the same order of magnitude are obtained if total cross sections are computed starting from the assumption that quarks are produced with a transverse momentum distribution similar to that of strongly interacting particles.

Another way of expressing our result is to state that the upper limit of the ratio quarks/ pions produced by $27.5-\mathrm{GeV} / c$ protons is of about $10^{-10}$.

The above limits are for stable quarks. If quarks are unstable, the cross-section limit must, of course, be increased depending on the lifetime and $Q$ value of the decay.

As previously stated, the negative result of this experiment refers to quarks with mass up to about $2.5 \mathrm{GeV}$, if these were produced in pairs on free nucleons at rest. If one considers 
the Fermi motions of the nucleons in the copper target, the limit on the quark mass can be appreciably increased, appropriately increasing, of course, the cross-section limit. Calculations by Gottfried, $\mathbf{3}$ assuming a Gaussian momentum distribution in the nucleus, indicate that there is an $18 \%$ probability for a nucleon in the nucleus to have a momentum greater than 1.5 times the Fermi momentum of $250 \mathrm{MeV} / c$ and a $0.5 \%$ probability for 2.5 times. Assuming instead a hard-sphere gas, the probabilities are $15 \%$ and $4 \%$ for 375 and $625 \mathrm{MeV} / c$, respectively. From a study of inelastic electronnucleus reactions, Ericson ${ }^{4}$ has estimated that there is a probability of about $0.5 \%$ for a nucleon to have a momentum of greater than 600 $\mathrm{MeV} / c$.

The head-on collision of the incoming proton with nucleons of 375 and $625 \mathrm{MeV} / c$ may lead to the production of quarks with mass up to 3.4 $\mathrm{GeV}$ and $4.0 \mathrm{GeV}$, respectively. Therefore, using the above probabilities and considering our acceptance angle, our experiments would set an upper limit of $\approx 10^{-33} \mathrm{~cm}^{2}$ and of $\approx 10^{-32}$ $\mathrm{cm}^{2}$ for these two masses.

Previous searches for fractionally charged particles, all unsuccessful, have been reported by Morrison, ${ }^{5}$ Bingham et al., ${ }^{6}$ and Leipuner et al. ${ }^{7}$ The first two experiments involved the scanning of existing bubble chamber film, for which there was no record of the time of arrival of the particles. The third work was a counter experiment which gave a total cross-section limit of $10^{-34} \mathrm{~cm}^{2}$ for quarks with mass up to $2 \mathrm{GeV}$.

We wish to thank Professor G. Cocconi, Pro- fessor B. Gregory, Professor P. Kabir, and Professor Ch. Peyrou for many helpful discussions. We are deeply indebted to the operating crews of the CERN proton synchrotron and of the Saclay $81-\mathrm{cm}$ hydrogen bubble chamber.

\footnotetext{
* Accepted without review under policy announced in Editorial of 20 July 1964 [Phys. Rev. Letters 13,79 (1964)].

†On leave from the Max-Planck Institute, Munich, Germany.

$\ddagger$ On leave from the Institute for Nuclear Research, Cracow, Poland.

$\S$ On leave from the University of Birmingham, Birmingham, Birmingham, England.

IOn leave from the Institute for Theoretical Physics, Vienna, Austria.

** On leave from the Imperial College, London, England.

†† On leave from the University of Oxford, Oxford, England.

${ }^{1}$ M. Gell-Mann, Phys. Letters 8,214 (1964).

${ }^{2}$ G. Zweig, CERN Report No. 8182/TH401, 1964 (unpublished), and to be published.

${ }^{3} \mathrm{~K}$. Gottfried, private communication.

${ }^{4}$ T. E. O. Ericson, private communication.

${ }^{5}$ D. R. O. Morrison, Phys. Letters 9 , 199 (1964). Here a different system of target operation makes the occurence of "early" tracks very improbable.

${ }^{6} \mathrm{H}$. H. Bingham, M. Dickinson, R. Diebold, W. Koch, D. W. G. Leith, M. Nikolić, B. Ronne, R. Huson, P. Musset, and J. J. Veillet, Phys. Letters $\underline{9}, 201$ (1964). In the heavy liquid chamber used here, the different conditions of operation and illumination make "early" tracks more distinguishable by the larger diameter of their images on the film.

${ }^{7}$ L. P. Leipuner, W. T. Chu, R. C. Larsen, and R. K. Adair, Phys. Rev. Letters 12, 423 (1964).
}

\section{NEUTRAL STRANGE-PARTICLE PRODUCTION AND $Y_{1}{ }^{*}(1385)$ FORMATION IN $p-p$ COLLISION AT $5.5 \mathrm{GeV} / c^{\dagger}$}

G. Alexander, O. Benary, N. Kidron,* A. Shapira, R. Yaari, and G. Yekutieli Department of Nuclear Physics, The Weizmann Institute of Science, Rehovoth, Israel (Received 18 August 1964)

This note presents results on the production of neutral strange particles, and the formation of the $Y_{1}{ }^{*}(1385)$ resonance in $p-p$ collisions at $5.52 \mathrm{GeV} / c .^{1}$ The study is based on 30000 pictures taken with the Saclay $81-\mathrm{cm}$ hydrogen bubble chamber exposed to a secondary 5.5$\mathrm{GeV} / c$ proton beam at CERN. Measurements on a sample of long beam tracks (larger than $50 \mathrm{~cm}$ ) gave an average beam momentum of $5.52 \pm 0.01 \mathrm{GeV} / c$. A total contamination of
$3.9 \%$ was found, of which about $1.5 \%$ was due to $K^{+}$and $2.4 \%$ to $\pi^{+}, \mu^{+}$, and $e^{+}$together. $25725 p-p$ events were found in an inscribed fiducial volume corresponding to a total path length of $1.67 \times 10^{7} \mathrm{~cm}$ and a total $p-p$ cross section $^{2}$ of $42.1 \pm 1.3 \mathrm{mb}^{3}$

A sample of $83 p-p$ events associated with either $\Lambda^{0}$ or $K_{1}{ }^{0}$ decays was found in the inscribed fiducial volume. Using the CERN programs THRESH and GRIND, the sample was subject to 